\title{
Comparison of Different Procedural Approaches for Left Atrial Appendage Closure: Searching for Simplicity
}

\author{
Rafael José Ruiz-Salmerón (D), ${ }^{1}$ Carlos Robles-Pérez ${ }^{D},{ }^{1}$ María Ronquillo-Japón,, \\ Rafael García-Borbolla, ${ }^{1}$ Manuel Iglesias-Blanco, ${ }^{1}$ Irene Méndez-Santos, ${ }^{1}$ \\ Carlos Rubio-Iglesias, ${ }^{1}$ Julia Ginsburg, ${ }^{1}$ Sergio Rodríguez-Leiras, ${ }^{1}$ \\ César Carrascosa-Rosillo, ${ }^{1}$ Manuel Vizcaíno-Arellano, ${ }^{1}$ and Juan Polo-Padillo ${ }^{2}{ }^{2}$ \\ ${ }^{1}$ Servicio Endovascular, Virgen Macarena University Hospital, Seville, Spain \\ ${ }^{2}$ Departamento de Medicina Preventiva y Salud Pública, Seville University, Seville, Spain
}

Correspondence should be addressed to Rafael José Ruiz-Salmerón; rjruizsalmeron@yahoo.es

Received 2 September 2020; Revised 9 January 2021; Accepted 12 January 2021; Published 25 January 2021

Academic Editor: Toshiko Nakai

Copyright (C) 2021 Rafael José Ruiz-Salmerón et al. This is an open access article distributed under the Creative Commons Attribution License, which permits unrestricted use, distribution, and reproduction in any medium, provided the original work is properly cited.

\begin{abstract}
Aim. To assess procedural and long-term efficacy and safety of two alternative methods for appendage closure, conscious sedation with standard transoesophageal echo and procedure guided by rotational angiography. Background. Demand for appendage closure is increasing, and a reasonable time-response should be given to nonvalvular atrial fibrillation patients not suitable to receive anticoagulation. General anesthesia and the need for an anesthesiologist are limiting factors to improve procedure availability; it is time to introduce simpler approaches. Methods. Single center experience in appendage closure during 9 years, using three different procedural approaches: general anesthesia with echo guidance, conscious sedation with echo guidance, and rotational angiography guidance. Conscious sedation and rotational angiography-guided procedures were performed in the absence of an anesthesiologist. Procedural characteristics and follow-up events were recorded. Results. 260 consecutive appendage closure procedures were reviewed: 155 were performed under general anesthesia (59.6\%), 71 were performed with conscious sedation $(27.3 \%)$, and 34 were rotational angiography guided $(13.1 \%)$. Device success rate for procedures guided by rotational angiography was significantly lower than that for general anesthesia and conscious sedation ( $91.2 \%$ versus $100 \%$ versus $98.6 \%$, $p=0.001$ ) because there was a greater need to recapture and change device size. However, final procedural success was high and without difference between approaches $(98.8 \%$ versus $97.2 \%$ versus $100 \%$, for general anesthesia, conscious sedation, and rotational angiography, respectively); with a median follow-up of 17 months (CI 95\% 13-23 month) (637.9 patients-year), there was no difference among approaches for thromboembolic (1.3 versus 1.8 versus 1.8 ) and major bleeding events (3.2 versus 2.8 versus 1.8), respectively. Conclusions. Appendage closure performed, either with conscious sedation with echo guidance or rotational angiography guided, is feasible, with no difference in procedural success and follow-up events compared with general anesthesia and without the limitation of the need for an anesthesiologist on-site.
\end{abstract}

\section{Introduction}

Left atrial appendage closure (LAAC) is the second most commonly performed structural procedure in Spain [1], 921 interventions in 2019. Nonetheless, LAAC indication is far from reaching its true potential, and less than $5 \%$ of possible candidates in Europe or United States actually benefit from this therapy [2].
As LAAC becomes more frequently used in our daily practice, there is a greater need to improve its availability, so that we may reduce the time at the risk of ischemic stroke or severe bleeding in patients with nonvalvular atrial fibrillation and contraindication for oral anticoagulation.

Historically, LAAC has usually been performed under general anesthesia, as general anesthesia guarantees the patient's tolerance to a prolonged transesophageal 
echocardiography (TOE) examination. It results in greater patient comfort and improved procedural safety. Nonetheless, it is generally assumed that general anesthesia is also one of the most limiting factors for LAAC availability as it requires the presence of an anesthesiologist. In an attempt to avoid the need for general anesthesia and to facilitate the performance of LAAC, other procedural approaches such as micro-TOE probe with conscious sedation [3], intracardiac echo [4], and even 3D computed tomography fusion images [5] have been described. Although feasible for LAAC guidance, these methods have limitations. Micro-TOE probe offers lower image quality than standard 3D echo probes. Intracardiac echo guidance generally requires advanced skills and increases procedural costs. Computed tomography studies are usually acquired at a different time and under different hemodynamic conditions than LAAC procedure.

The difficulty to have anesthesia availability for every LAAC procedure motivated us to evaluate new procedural approaches in the absence of an anesthesiologist. Conscious sedation with LAAC guided by TOE or the procedure guided by rotational angiography with patient awake and collaborative was performed. The procedural and long-term results of these two alternative LAAC approaches were compared to LAAC performed under general anesthesia. The aim was to determine if these new approaches could maintain similar efficacy and safety as that obtained by the standard LAAC under general anesthesia.

\section{Materials and Methods}

This study reports our LAAC experience, performed in a single center, and the same team over a 9 year period, March 2011 to February 2020. Prior to each LAAC procedure, the patient signed an informed consent. Baseline clinical characteristics, procedural variables, and 7-day events were initially recorded. Follow-up assessment was performed on February 2020 by consulting our digital medical record platform, DIRAYA ${ }^{\mathrm{TM}}$ for the Andalusian Healthcare System, Seville, Spain.

Upon its inception, LAAC incorporated general anesthesia and TOE guidance (Acuson X300 and Acuson SC2000, Siemens, Germany). After 2.5 years, a new approach was needed because the patient case load was too great to depend upon the presence of an anesthesiologist for every single case. A conscious sedation protocol was adopted and performed by our own staff, consisting of a dual drug regimen, meperidine (50 $\mathrm{mg}$ bolus) and propofol (initial perfusion of $0.5 \mathrm{mg} / \mathrm{kg}$ in 3 minutes, followed by a bolus of $10 \mathrm{mg}$ as needed to maintain the patient's tolerance to the probe). The procedure was guided by standard TOE, and the same probe was used as with the general anesthesia approach.

LAAC guided by rotational angiography was adopted 4 years after initiating our program, and it was indicated in the case of TOE contraindication. During this approach, the patient was awake and maintained a fixed position. Only for this group, cardiac computed tomography was recommended 24-48 hours prior to the intervention in order to exclude thrombus inside the left appendage. After transeptal puncture, the protocol consisted of an initial angiography from the exterior of the left appendage to confirm that it was free of thrombus (this maneuver become crucial importance in case of absence of a recent computed tomography study). Following appendage cannulation with a pigtail, a 3D image was reconstructed by $180^{\circ}$ rotational acquisition (dye volume of $48 \mathrm{ml}$, with infusion rate of $8 \mathrm{ml} / \mathrm{sec}$ ), and it was fused with fluoroscopic image (i-Pilot ${ }^{\mathrm{TM}}$, Siemens, Germany) (Figure 1).

Each of the three LAAC approaches was chosen based upon logistical and clinical circumstances; whenever anesthesia was available, then the procedures were performed under general anesthesia. Otherwise, conscious sedation was utilized. Nonetheless, when esophageal disease prevented the use of a TOE probe, then rotational angiography approach was selected.

Three different types of devices were implanted: Watchman $^{\mathrm{TM}}$ (gen 2, 5, and Watchman Flex) (Boston Scientific, USA), Amplatzer ${ }^{\mathrm{TM}}$ ACP/Amulet (Abbott, USA), and LAmbre $^{\mathrm{TM}}$ (Lifetech Scientific, China). The latter was implanted in very few cases. There were no anatomical or clinical driven criteria to select one device over another; in fact, the strategy was to balance the volume of use of each one. As an exception, Amulet ${ }^{\mathrm{TM}}$ was preferred for procedures guided exclusively by rotational angiography, as its release criteria were mainly based on fluoroscopic images.

LAAC success was measured by two criteria: device success (successful deployment of the first selected device) and procedural success (final successful deployment, free of adverse events within the first 24 hours postprocedure). Standard echocardiography and fluoroscopy criteria were followed for device release; for cases performed by rotational angiography guidance, device release decision was based on its position and stability assessment on 3D rendered fusion image after a final angiography with the simultaneous tugtest.

Long-term follow-up events were assessed: cardiovascular death, thromboembolic (ischemic stroke/systemic embolism), and major bleeding (as defined by the HASBLED validation: intracranial bleeding, hospitalization, and a hemoglobin fall greater than $2 \mathrm{~g} / \mathrm{L}$ or need for transfusion). LAAC clinical effectiveness was assessed by the percentage of relative risk reduction of ischemic stroke/systemic embolism and of major bleeding at follow-up. The estimated risks were calculated by $\mathrm{CHA}_{2} \mathrm{DS}_{2} \mathrm{VASc}$ and HASBLED scores, respectively.

2.1. Statistical Analysis. The calculations were performed using the IBM SPSS v26.0 and Epidat 4.2 programs and initially consisted of a descriptive analysis of the data, generating mean and standard deviations for numeric variables, and frequency distributions and percentages for categorical variables.

The comparison between demographic and clinical quantitative variables was made by the ANOVA test, checking hypotheses of normality with the Shapiro-Wilk test; in case of a significant difference, multiple comparisons were made using the Bonferroni test. 


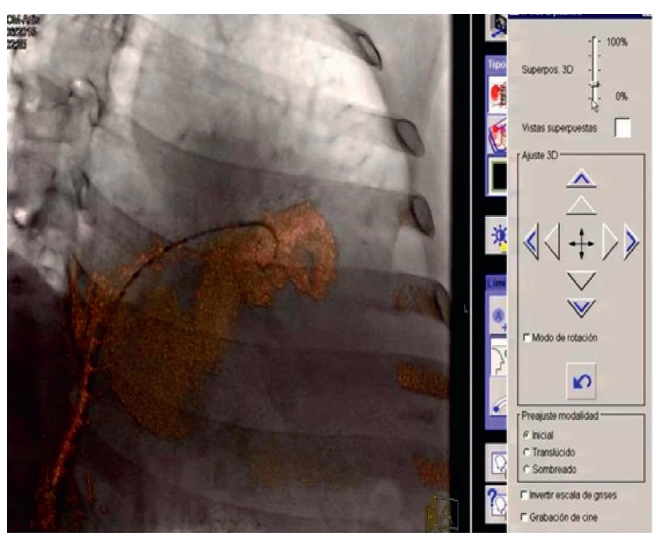

(a)

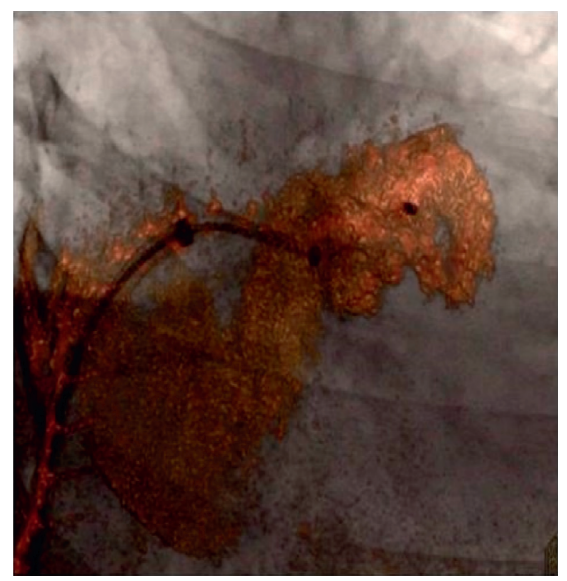

(c)

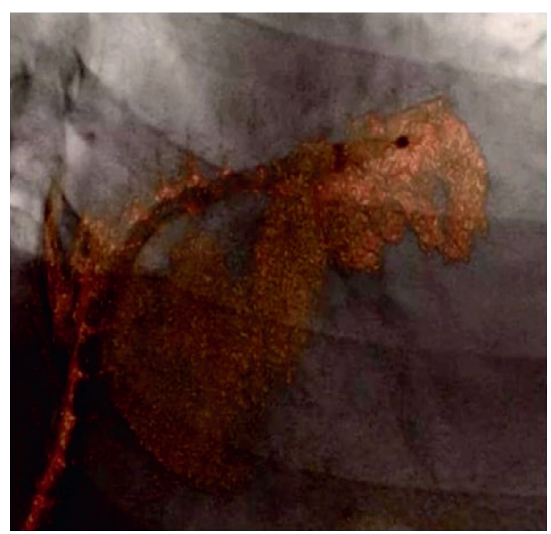

(b)

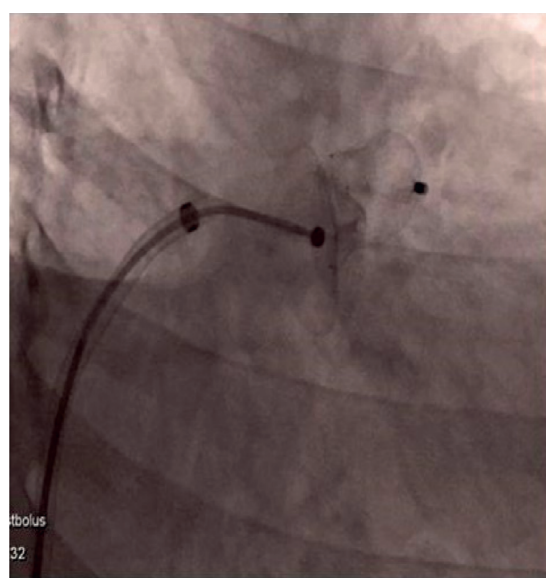

(d)

Figure 1: LAAC guided by rotational angiography. (a) Syngo i-Pilot ${ }^{\circledR}$ (Siemens, Germany) software allows the generation of the left atrial appendage mask by tomographic rotational acquisition during intraappendage angiography. Appendage mask is superimposed to fluoroscopic images, changing its shape as the projection changes. (b) Appendage cannulation with Amplatzer sheath and initiation of Amulet device deployment. (c) and (d) Final Amulet deployment with (c) and without (d) superimposed mask on fluoroscopy.

The comparison of qualitative variables was made using contingency tables and the chi-squared test.

Finally, the comparison between event incidence rates was made using the Rothman test, and confidence intervals were calculated by the Rosner method.

\section{Results}

3.1. Clinical Characteristics of Our Population. Our population consisted of 260 patients with nonvalvular atrial fibrillation between 42 and 92 years and postprocedure follow-up ranged from 1 to 107 months. Patients were distributed among the three different procedural approaches: 155 general anesthesia (59.6\%), 71 conscious sedation $(27.3 \%)$, and 34 guided by rotational angiography $(13.1 \%)$.

In general, the main indication for LAAC was the contraindication to oral anticoagulation either absolute due to a bleeding event (229 patients, $88.1 \%$ ) or relative because of underlying bleeding risk pathology (1.1\%), brain tumor (2 patients) and aorta dissection (1 patient). For the remaining
28 patients $(10.8 \%)$, indication was the inability to take oral anticoagulation for a reason other than high risk for bleeding (13 patients refused anticoagulant treatment, 12 patients were unable to take anticoagulants due to psychiatric illness, suboptimal INR control, increased risk of falls, or thromboembolic event despite optimal anticoagulation, and 3 patients with ischemic stroke). Rotational angiography approach was utilized more frequently in patients without contraindication to oral anticoagulation, compared to general anesthesia and conscious sedation $(26.5 \%$ versus $8.4 \%$ and $12.7 \%$, respectively, $p=0.08$ ).

Treatment at time of LACC indication was also different among the approaches. The majority of the global population was anticoagulated (68.8\%), and this was also the case for general anesthesia (73.6\%) and conscious sedation (69\%). Nevertheless, among patients with LAAC guided by rotational angiography, mostly $53 \%$ was without anticoagulation $(p<0.001)$.

Other clinical characteristics, also the $\mathrm{CHA}_{2} \mathrm{DS}_{2} \mathrm{VASc}$ and HASBLED scores, were not different among the three LAAC approaches (Table 1). 
TABLE 1: Baseline clinical characteristics at time of LAAC indication.

\begin{tabular}{|c|c|c|c|c|}
\hline & Global population & General anesthesia & Conscious sedation & Angiography guided \\
\hline Age, years $($ mean $\pm S D)$ & $74.8 \pm 8.1$ & $74.2 \pm 8.0$ & $75.2 \pm 8.0$ & $77.1 \pm 8.9$ \\
\hline Male, $n(\%)$ & $160(61.5 \%)$ & $97(62.6 \%)$ & $40(56.3 \%)$ & $23(67.6 \%)$ \\
\hline Hypertension, $n(\%)$ & $238(91.5 \%)$ & $142(91.6 \%)$ & $68(95.8 \%)$ & $28(82.4 \%)$ \\
\hline Diabetes, $n(\%)$ & $118(45.4 \%)$ & $75(48.4 \%)$ & $26(36.6 \%)$ & $17(50.0 \%)$ \\
\hline Dyslipidemia, $n(\%)$ & $130(50 \%)$ & $80(51.6 \%)$ & $30(42.3 \%)$ & $20(58.8 \%)$ \\
\hline Smoker, $n(\%)$ & $93(35.8 \%)$ & $61(39.4 \%)$ & $18(25.4 \%)$ & $14(41.2 \%)$ \\
\hline Renal insufficiency, $n(\%)$ & $64(24.6 \%)$ & $42(27.1 \%)$ & $14(20.0 \%)$ & $8(23.5 \%)$ \\
\hline Ischemic cardiopathy, $n(\%)$ & $89(34.2 \%)$ & $58(37.4 \%)$ & $22(31.4 \%)$ & $9(26.5 \%)$ \\
\hline \multicolumn{5}{|l|}{ Stroke, $n(\%)$} \\
\hline Ischemic stroke & $38(14.6 \%)$ & $23(14.8 \%)$ & $10(14.1 \%)$ & $5(14.7 \%)$ \\
\hline Haemorrhagic stroke & $57(21.9 \%)$ & $36(23.2 \%)$ & $15(21.1 \%)$ & $6(17.6 \%)$ \\
\hline \multicolumn{5}{|l|}{ Treatment before LAAC, $n(\%)\left({ }^{*}\right)$} \\
\hline No & $33(12.7 \%)$ & $16(10.3 \%)$ & $6(8.5 \%)$ & $11(32.4 \%)$ \\
\hline Antiaggregation & $48(18.5 \%)$ & $25(16.1 \%)$ & $16(22.5 \%)$ & $7(20.6 \%)$ \\
\hline Anti-K & $83(31.9 \%)$ & $44(28.4 \%)$ & $34(47.9 \%)$ & $5(14.7 \%)$ \\
\hline NOAC & $71(27.3 \%)$ & $50(32.3 \%)$ & $13(18.3 \%)$ & $8(23.5 \%)$ \\
\hline Combined & $25(9.6 \%)$ & $20(12.9 \%)$ & $2(2.8 \%)$ & $3(8.8 \%)$ \\
\hline \multicolumn{5}{|l|}{ Indication, $n(\%)(\$)$} \\
\hline GI bleeding & $93(35.8 \%)$ & $58(37.4 \%)$ & $22(31.0 \%)$ & $13(38.2 \%)$ \\
\hline Cerebral bleeding & $58(22.3 \%)$ & $37(23.9 \%)$ & $15(21.1 \%)$ & $6(17.6 \%)$ \\
\hline Other bleeding & $78(30.0 \%)$ & $47(30.3 \%)$ & $25(35.2 \%)$ & $6(17.6 \%)$ \\
\hline Elective/others & $31(11.9 \%)$ & $13(8.4 \%)$ & $9(12.7 \%)$ & $9(26.5 \%)$ \\
\hline $\mathrm{CHA}_{2} \mathrm{DS}_{2} \mathrm{VASc}($ mean $\pm \mathrm{SD})$ & $4.3 \pm 1.6$ & $4.4 \pm 1.7$ & $4.1 \pm 1.6$ & $4.6 \pm 1.5$ \\
\hline $\operatorname{HASBLED}($ mean $\pm \mathrm{SD})$ & $3.7 \pm 1.2$ & $3.7 \pm 1.3$ & $3.8 \pm 1.2$ & $3.6 \pm 1.0$ \\
\hline
\end{tabular}

$\left(^{*}\right) p<0.001 ;(\$) p=0.08$.

3.2. Procedural Characteristics and LAAC Success. Two versions of Watchman ${ }^{\mathrm{TM}}$ were deployed, gen 2.5 (125 patients) and Flex (17 patients), and also two versions of Amplatzer $^{\mathrm{TM}}$ (ACP (16 patients) and Amulet (100 patients)). LAmbre $^{\mathrm{TM}}$ was implanted in only two patients.

Different approaches involved differences in procedural variables such as fluoroscopic time and dose of radiation. The general anesthesia approach was the longest, whereas conscious sedation was the shortest $(15.7 \pm 11.5$ minutes versus $12.2 \pm 4.2$ minutes, respectively, $p=0.04)$. Rotational angiography guidance incurred the highest radiation dose, while the conscious sedation incurred the lowest $\left(24299 \pm 14278 \mu \mathrm{Gym}^{2}\right.$ versus $16148 \pm 458 \mu \mathrm{Gym}^{2}$, respectively, $p=0.03)$.

Device success was $98.5 \%$ for the global population. Device failure occurred in 4 patients, and it was due to the implantation of a second device after total recapture of the first choice, in three cases due to change of size and in one case due to both the change of type and size of the device. Device success rate for rotational angiography-guided procedures was significantly lower than that for general anesthesia and conscious sedation (91.2\% versus $100 \%$ versus $98.6 \%, p=0.001$ ).

Procedural success was achieved in $98.8 \%$ of the population. Different approaches did not show major differences in procedural success rate: $99.4 \%$ for general anesthesia (1 case of major bleeding during tracheal intubation), $97.2 \%$ for conscious sedation ( 2 failed cases due to cardiac tamponade), and $100 \%$ for rotational angiography guidance.
Neither deaths nor ischemic strokes or systemic embolisms were reported during or within the first 24 hours of the procedures.

Adverse events within the first 7 days after the procedure consisted of 6 complications (2.3\%): 3 events (1.9\%) for the general anesthesia and 3 events (4.2\%) for the conscious sedation group. Adverse events are summarized in Table 2.

Median follow-up was 17 months (CI 95\% 13-23 month) (637.9 patient-year). Cardiovascular death, thromboembolic events (ischemic stroke and systemic embolism), and major bleeding rates (100 patient-year) did not show significant differences among approaches (Table 3 and Figure 2).

\section{Discussion}

LAAC is a mature therapy for preventing thromboembolic events in patients with nonvalvular atrial fibrillation and contraindication for oral anticoagulation, supported by solid clinical data [6-8]. To date, LAAC utilization is not being indicated to its full potential; more than one million European and 660 thousand North American patients with nonvalvular atrial fibrillation are potential candidates for LAAC [2].

At the inception of LAAC and due to initial safety issues, the procedure was performed following strict protocol with TOE guidance, usually under general anesthesia. General anesthesia facilitates a comfortable experience for the patient and provides a complete TOE imaging assessment, increasing procedure safety. 
TABLE 2: Adverse events during first 7 days.

\begin{tabular}{lcccc}
\hline Day & Adverse event & Description & Death & Approach \\
\hline Procedure & Bleeding & Orotracheal intubation & No & General anesthesia \\
Procedure & Tamponade & Pericardiocentesis & No & Conscious sedation \\
1 day & Tamponade & Pulmonary artery erosion. Surgery & Conscious sedation \\
4 day & Bronchoaspiration & Bronchoaspiration while eating & Conscious sedation \\
4 day & Bleeding & Upper digestive track bleeding & Yes & General anesthesia \\
6 day & Bleeding & Upper digestive track bleeding & No & General anesthesia \\
\hline
\end{tabular}

TABLE 3: Follow-up events. Comparison among approaches for cardiovascular death, ischemic stroke/systemic embolism, and major bleeding.

\begin{tabular}{lcccc}
\hline & Patient-year & $\begin{array}{c}\text { Cardiovascular death } \\
(100 \text { patient-year })\end{array}$ & $\begin{array}{c}\text { Ischemic stroke/Systemic embolism } \\
\text { (100 patient-year) }\end{array}$ & Major bleeding (100 patient-year) \\
\hline General anesthesia & 472.2 & 4.9 & 1.3 & 3.2 \\
Conscious sedation & 108.8 & 2.8 & 1.8 & 2.8 \\
Rotational angiography & 56.9 & 5.3 & 1.8 & 1.8 \\
\hline
\end{tabular}

Cardiovascular death, general anesthesia vs. conscious sedation (relative risk (RR 1.8 (CI 95\% 0.5-9.2), $p=0.3$ ); general anesthesia vs. rotational angiography guidance (RR 0.9 (CI 95\% 0.3-5.0), $p=0.9$ ); conscious sedation vs. rotational angiography guidance (RR 0.5 (CI 95\% 0.1-4.0), $p=0.5$ ).

Ischemic stroke/systemic embolism, general anesthesia vs. conscious sedation (RR 0.7 (CI 95\% 0.1-33.3), $p=0.7$ ); general anesthesia vs. rotational angiography guidance (RR 0.8 (CI 95\% 0.1-34.8), $p=0.7$ ); conscious sedation vs. rotational angiography guidance (RR 0.8 (CI 95\% 0.1-6.2), $p=0.8$ ).

Major bleeding, general anesthesia vs. conscious sedation (RR 1.1 (CI 95\% 0.3-6.2), $p=0.9$ ); general anesthesia vs. rotational angiography guidance (RR 1.8 (CI 95\% 0.3-76.1), $p=0.6$ ); conscious sedation vs. rotational angiography guidance (RR 1.8 (CI 95\% 0.2-13.7), $p=0.6$ ).

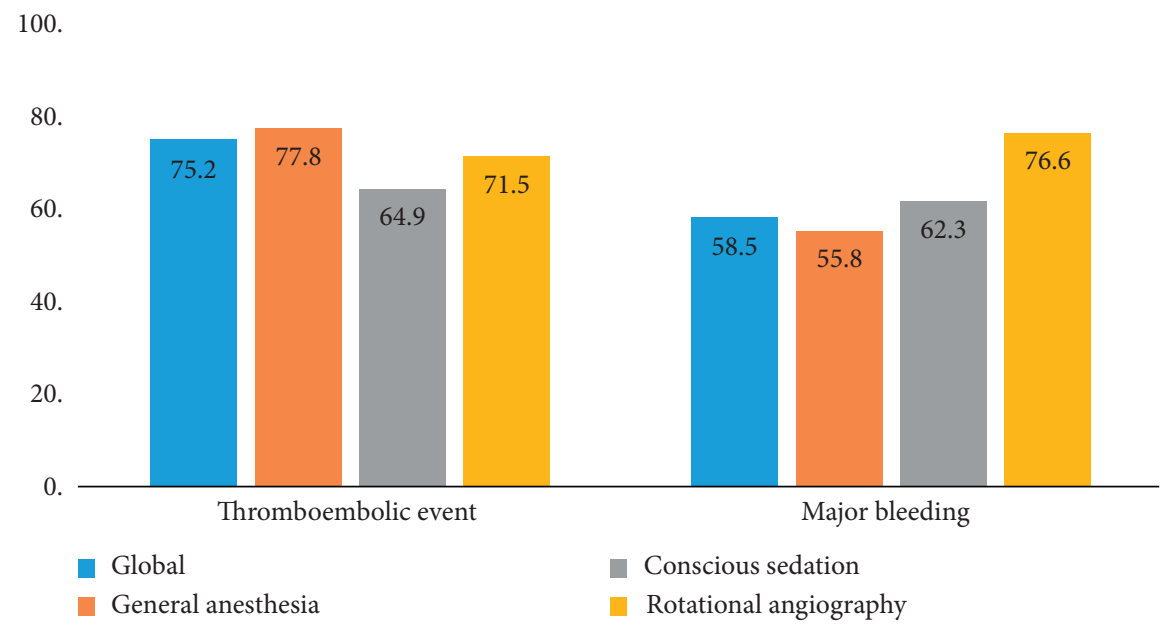

FIGURE 2: Relative risk reduction (percentage) for thromboembolic events (ischemic stroke/systemic embolism) and for major bleeding in global population and for each LAAC approach.

The requirement to incorporate general anesthesia for each LAAC procedure when the indication volume increases has a countering effect; it reduces LAAC availability thus leaving potential candidates at a higher risk because of the inability to perform the procedure when it is truly needed. Therefore, it is reasonable that centers with greater LAAC volume and experience should explore different options to expand LAAC availability. LAAC procedures performed without general anesthesia have been published, either with micro-TOE imaging under conscious sedation or other imaging modalities [3-5].
In contrary to TAVI and other structural procedures, the debate to use conscious sedation versus general anesthesia has yet to reach LAAC. Conscious sedation could be performed by specialists other than anesthesiologists, thus increasing LAAC availability and reducing time to intervention for a patient at clinical crossroads. LAAC under conscious sedation has been a reality for us since 2013 and has been applied whenever an anesthesiologist has not been available for the procedure. Conscious sedation has been performed by our staff through the application of a simple protocol, and no case of patient complication or intolerance 
to the standard TOE probe was recorded. Therefore, this downplays the debate to use a micro-TOE probe versus a standard probe. A third approach, LAAC under rotational angiography guidance, was reserved for a minority of cases when TOE was contraindicated.

In our experience, LAAC under conscious sedation and guided with TOE was the approach with the shortest fluoroscopic time and the lowest dose of radiation. The procedure was probably shorter because the operator sped up the intervention so as to reduce potential patient discomfort. It is not surprising that LAAC guided by rotational angiography exposed the patient and the operator to a higher dose of radiation due to tomographic $3 \mathrm{D}$ reconstruction of the appendage.

LAAC guided by rotational angiography yielded the lowest device success rate. This highlights the value of TOE imaging to double-check appendage measurements; however, procedural success rates were high and homogeneous, and there were no differences between approaches with or without general anesthesia or between TOE and no TOE guidance. This is of upmost importance because a center with LAAC experience could assume all three procedural approaches to guarantee LAAC availability without compromising safety. In fact, the rate of adverse events within the first seven days after the procedure ranged from $1.9 \%$ to $4.2 \%$ (Table 2 ) versus published reports of $2.8 \%$ to $8 \%$ [9]. There was a bleeding complication directly related to general anesthesia and a tamponade in a patient under conscious sedation. Remaining complications having occurred between days 1 and 7 probably had nothing to do with the LAAC approach.

Equally, if not more important, the procedural results are the long-term clinical benefits after LAAC. It is crucial to analyze if procedural approaches, other than the standard, could lead to a reduced clinical benefit at followup. After a median time of 17 months (CI 95\% 13-23 month), the LAAC procedure performed, either under conscious sedation with TOE or under rotational angiography, did not show any difference in long-term clinical benefit parameters compared to the standard general anesthesia with TOE. More specifically, cardiovascular deaths, thromboembolic events, and major bleeding episodes were not significantly different among the three approaches.

Finally, although these procedural approaches were performed prior to the Coronavirus pandemic, it is evident that this new scenario further motivates us to minimize invasive techniques and especially aerosol generating procedures such as tracheal intubation [10].

There are several limitations of this study. In addition to being a retrospective study, the main limitation is the small population of the rotational angiography guidance group because it was reserved to those with TOE contraindication.

\section{Conclusion}

LAAC performed under conscious sedation or guided by rotational angiography are valid and attractive approaches when considering both short-term procedural results and long-term clinical benefits. In comparison with general anesthesia, the new approaches increase LAAC availability and allow an autonomous programming of the procedures without the presence of an anesthesiologist, that means, a faster response time to a greater number of patients.

\section{Data Availability}

The data used to support the findings of this study are obtained from DIRAYA ${ }^{\mathrm{TM}}$ for the Andalusian Healthcare System, Seville, Spain.

\section{Conflicts of Interest}

The authors declare that they have no conflicts of interest.

\section{Acknowledgments}

The authors would like to acknowledge Richard A. Ginsburg for providing language assistance.

\section{References}

[1] S. Ojeda, R. Romaguera, I. Cruz-González, and R. Moreno, "Registro español de Hemodinámica y cardiología intervencionista. XXIX informe oficial de la Asociación de Cardiología intervencionista de la Sociedad española de Cardiología (1990-2019)," Revista Española de Cardiología, vol. 73, no. 11, pp. 927-936, 2020.

[2] M. Fukutomi, O. De Backer, and L. Søndergaard, "Indications, current adoption and future perspectives for percutaneous left atrial appendage closure," EuroIntervention, vol. 14, no. 17, pp. 1707-1709, 2019.

[3] G. Jiménez Brítez, L. Sanchís, A. Regueiro, M. Sabaté, M. Sitges, and X. Freixa, "Minimally-invasive transesophageal echocardiography for left atrial appendage occlusion with a latest-generation microprobe. Initial experience," Revista Española de Cardiología (English Edition), vol. 72, no. 6, pp. 511-512, 2019.

[4] J. Saw, "Intracardiac echocardiography for endovascular left atrial appendage closure," JACC: Cardiovascular Interventions, vol. 10, no. 21, pp. 2207-2210, 2017.

[5] A. K. Roy, J. Horvilleur, B. Cormier et al., "Novel integrated 3D multidetector computed tomography and fluoroscopy fusion for left atrial appendage occlusion procedures," Catheterization and Cardiovascular Interventions, vol. 91, no. 2, pp. 322-329, 2018.

[6] V. Y. Reddy, S. K. Doshi, S. Kar et al., "5-Year outcomes after left atrial appendage closure," Journal of the American College of Cardiology, vol. 70, no. 24, pp. 2964-2975, 2017.

[7] L. V. A. Boersma, B. Schmidt, T. R. Betts et al., "Implant success and safety of left atrial appendage closure with the WATCHMAN device: peri-procedural outcomes from the evolution registry," European Heart Journal, vol. 37, no. 31, pp. 2465-2474, 2016.

[8] U. Landmesser, C. Tondo, J. Camm et al., "Left atrial appendage occlusion with the AMPLATZER Amulet device: one-year follow-up from the prospective global Amulet observational registry," EuroIntervention, vol. 14, no. 5, pp. e590-e597, 2018. 
[9] M. Glikson, R. Wolff, G. Hindricks et al., "EHRA/EAPCI expert consensus statement on catheter-based left atrial appendage occlusion-an update," Eurointervention, vol. 15, no. 13, pp. 1133-1180, 2020.

[10] K. Tran, K. Cimon, M. Severn, C. L. Pessoa-Silva, and J. Conly, "Aerosol generating procedures and risk of transmission of acute respiratory infections to healthcare workers: a systematic review," PLoS One, vol. 7, Article ID e35797, 2012. 